\title{
Revealing the Surface Effect at Atomic Scale in Silver Hollandite
}

Xiaobing $\mathrm{Hu}^{1}$, Lijun $\mathrm{Wu}^{1}$, Yimei Zhu ${ }^{1}$, Jianping Huang ${ }^{2}$, Amy C. Marschilok ${ }^{2,3,4}$, Esther S. Takeuchi ${ }^{2,3,4}$ and Kenneth J. Takeuchi ${ }^{2,3}$

1. Condensed Matter Physics and Materials Science Department, Brookhaven National Laboratory, Upton, New York, USA.

2. Department of Chemistry, Stony Brook University, Stony Brook, New York, USA.

3. Department of Materials Science and Chemical Engineering, Stony Brook University, Stony Brook, New York, USA.

4. Energy Sciences Directorate, Brookhaven National Laboratory, Upton, New York, USA.

Hollandite based manganese oxides recently attracted much attention because of their large tunnel size to facilitate topological ionic intercalation [1]. Hollandite is widely recognized as an intrinsic 1D diffusion material, in which ions diffuse along the [001] tunneled direction. Interestingly, besides the 1D ionic diffusion, operando lithiation experiments also demonstrate ionic transport within the $a-b$ plane [2]. However, the underlying mechanisms remain elusive. In this study, we reveal the surface effect and discuss its role on ionic diffusion.

We study the silver hollandite nanorods as battery electrodes $\left(\mathrm{Li} / \mathrm{Ag}_{\mathrm{x}} \mathrm{Mn}_{8} \mathrm{O}_{16}\right)$. As shown in Fig. $1 \mathrm{a}$, the surface structure is different from the interior within individual rod. Although the basic crystal structure of the surface is the same as the interior, structural inhomogeneity exists within the (010) plane in the surface region as evidenced by the diffuse $\{020\}$ reflections along [010] direction (Fig. 1b). In addition, interplanar distance of $(020)$ at surface $(\sim 5.12 \AA)$ is $\sim 5.6 \%$ larger than that of the interior regions $(\sim$ $4.85 \AA$ ). Figs. $1 \mathrm{~d}$ and $1 \mathrm{e}$ are magnified images corresponding to the framed regions (1) and (3) indicated in Fig. 1a. For simplification, we only mark the positions of Ag and Mn layers in Fig. 1e based on the structural projection shown in Fig. 1f. The labels $\Delta_{1}$ and $\Delta_{2}$ in Fig. 1e represent the interlayer distance of neighboring $\mathrm{Mn}-\mathrm{Mn}$ and $\mathrm{Mn}-\mathrm{Ag}-\mathrm{Mn}$ columns, respectively. Integrated intensity profiles of regions (1), (2) and (3) are compared in Fig. 1h. It is found that at the very edge (indicated by (1)), there is almost none signal at Ag positions; while deviated from the very edge, Ag signal becomes stronger as shown in profiles (2) and (3). In addition, the distance of $\Delta_{1}$ gradually decreases from $2.6 \AA$ for the very edge region (1) to $1.8 \AA$ for the near surface region (2) and finally to $1.6 \AA$ for interior region; while the distance of $\Delta_{2}$ gradually increase from $2.6 \AA$ for region (1) to $3.0 \AA$ for region (2) and finally to $3.2 \AA$ for region (3). The variation of the lattice parameters $b$ equaling to $2 \cdot\left(\Delta_{1}+\Delta_{2}\right)$ and related lattice strain from surface to interior are shown in Fig. 1i. The measured lattice distance $b$ for interior is around $9.7 \AA$. However, this value greatly deviates from $9.7 \AA$ when approaching the surface. Specifically, for the first unit cell at the very edge, the lattice parameter $b$ is as large as $10.7 \AA$ with the lattice expansion around $10 \%$ in contrast to the interior region. More detailed measurements of the interlayer distance of $\Delta_{l}$ and $\Delta_{2}$ from the very edge to interior (based on Fig. 1a) is shown Fig. 1j. There is an obviously gradual of shrinkage (expansion) of interlayer distance $\Delta_{l}\left(\Delta_{2}\right)$ from edge to interior within a single rod.

The observed local structural modifications at atomic scale in hollandite result from the existence of $\mathrm{O}$ vacancies. The roles of surface $\mathrm{O}$ vacancies on ionic transport are further unraveled by DFT calculations. We demonstrate that $\mathrm{O}$ vacancies and atomic disorder at the surface regions can be valuable to create additional diffusion pathways, thus facilitating ionic transport in 1D nanomaterials [3]. 
References:

[1] Y Shao-Horn et al, J. Electrochem. Soc. 145 (1998), p. 582.

[2] F Xu et al, Nat. Commun. 8 (2017), p. 15400.

[3] This work was supported by the US DOE-BES, Materials Science and Engineering Division, under Contract No. DESC0012704 and the Center for Mesoscale Transport Properties under award \#DE-

SC0012673.
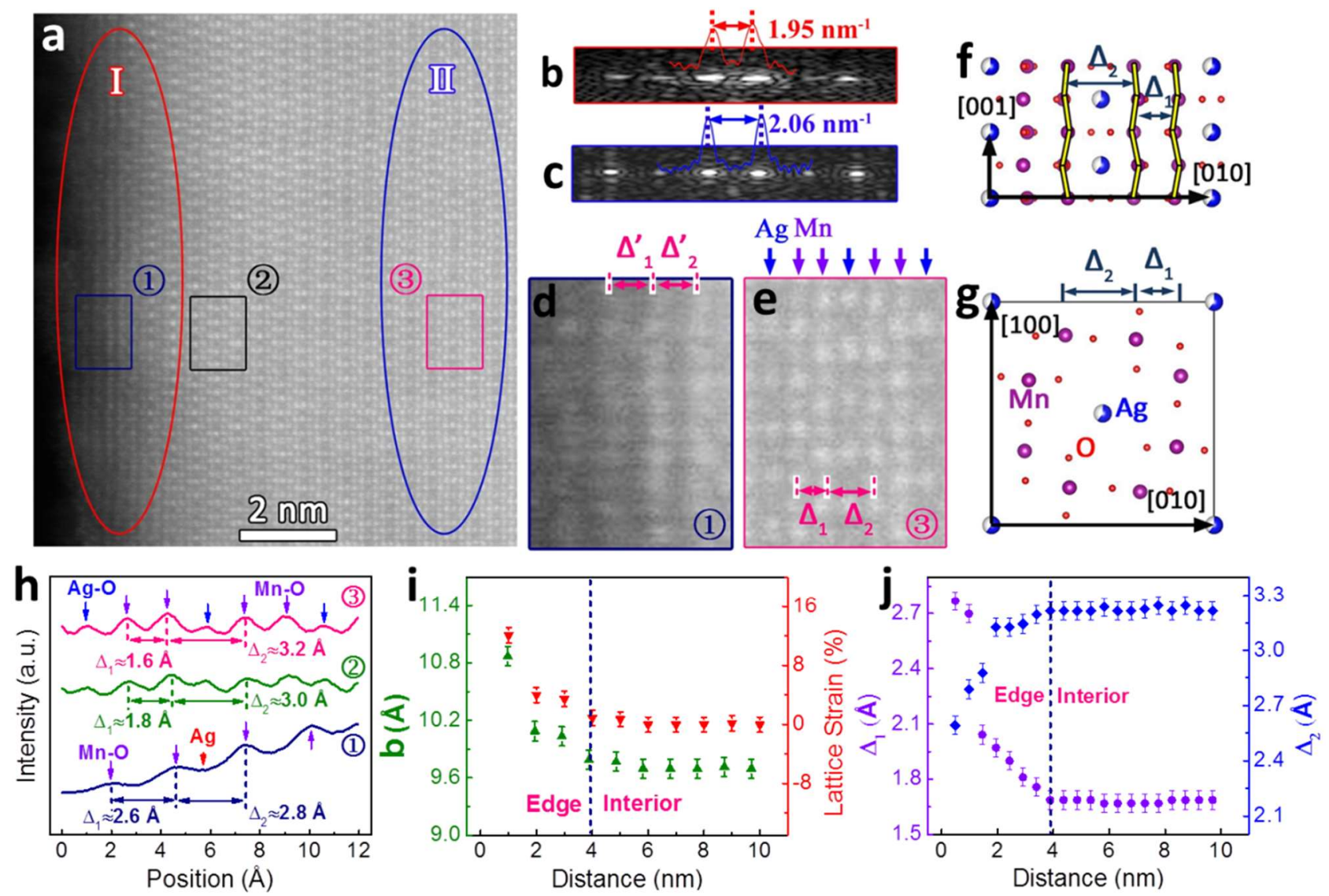

Figure 1. (a) Atomic resolution HAADF image along [100] zone-axis showing the structural features of a single nano-rod obtained from silver hollandite. (b, c) A row of FFT patterns corresponding to regions I and II in (a) respectively. The corresponding line scans of two neighboring spots are overlaid. The broadening of the Bragg peaks in (b) indicates the increased lattice distortion in the surface area (region I in (a)). (d, e) Magnified region (1) and (3) in (a). The solid arrows indicate the Ag and Mn layers. $\Delta_{l}$ and $\Delta_{2}$ represent the averaged interlayer distance of the neighboring $\mathrm{Mn}-\mathrm{Mn}$ and $\mathrm{Mn}-\mathrm{Ag}-\mathrm{Mn}$ layers respectively. (f, g) Structural projections of ideal silver hollandite viewing along [100] and [001] directions. The $\mathrm{Mn}, \mathrm{O}$ and partially occupied $\mathrm{Ag}$ cites are indicated. (h) The projected integrated intensity for region (1), 2), (3) in (a). (i) Lattice parameter $b$, and strain and (j) $\Delta_{1}, \Delta_{2}$ variations from edge to interior based on the image shown in (a). The strain in (i) is defined as $\Delta b / b$. 